\title{
NEUROPSYCHIATRIC FINDINGS IN PATIENTS EXPOSED TO POLYBROMINATED BIPHENYLS
}

\author{
Jeoffrey K. Stross \\ University of Michigan Medical School \\ Ann Arbor, Michigan 48109 \\ Robert K. Nixon and Marvin D. Anderson \\ Henry Ford Hospital \\ Detroit, Michigan 48201
}

\section{INTRODUCTION}

The inadvertent mixing of polybrominated biphenyls (PBBs) into animal feed in 1973 resulted in the contamination of thousands of Michigan farms. Farm animals who ingested this feed suffered various consequences including weight loss, decreased productivity, frequent infections, skin changes, and reproductive abnormalities. ${ }^{\prime}$ This error was not initially known, and widespread contamination of humans occurred through the consumption of animals and animal products. Families from quarantined farms were thought to have the heaviest exposure, with consumers of products from quarantined farms and chemical workers directly associated with PBBs also receiving heavy exposure.

At the time of this incident, little was known about the possible health effects of PBBs in humans. The only related experience was an accidental ingestion of polychlorinated biphenyls (PCBs) that occurred in Japan and caused many health problems. ${ }^{2-3}$

In Michigan, practicing physicians reported adverse health effects in patients, and the Department of Public Health initiated a study in 1974 to try to document these effects. This study failed to identify any acute effects on health, and neither a definite dose-response relationship nor a pattern of illness consistent with the extent of PBB exposure was identified. ${ }^{4}$ Because of a concern for delayed or late consequences of PBB ingestion, the Michigan Department of Public Health, in collaboration with several agencies of the Department of Health, Education, and Welfare initiated a multi-year comprehensive study of the possible human health consequences of exposure to PBBs. This study began in 1976 and historical data and blood specimens were collected and analyzed. Although data analysis is not complete, there appears to be no relationship between PBB serum levels and the presence or absence of symptoms. In fact, those people with no measurable PBBs in their serum reported the highest prevalence of health care complaints. ${ }^{5}$ Many of these complaints were neurobehavioral in nature and included poor memory, difficulty in concentration, personality changes, irritability, and hypersomnolence. Since laboratory studies in cattle revealed low PBB concentrations in the central nervous system, ${ }^{6}$ the role of PBB ingestion in the etiology of these complaints was in question. A recent study of farmers exposed to PBBs has shown a statistically significant increase in neurobehavioral complaints, as well as poorer performance in various tests of neurologic function as compared to controls. These results were also correlated with serum PBB levels. ${ }^{7}$ Since these findings were at odds with each other, this study was designed to evaluate these neurobehavioral complaints and this paper describes the findings. 


\section{METHODS AND MATERIALS}

Sixty-four persons with known exposure to PBBs and incapacitating health complaints were identified from previous studies. These people complained of a serious deterioration in their health status and were unable to engage in their previous occupations. They were contacted by letter and asked to participate in a research project aimed at determining the health effects of PBBs. Fifty persons agreed to participate, and 46 actually were evaluated in the study, 37 men and 9 women. Patients were evaluated in the Clinical Research Center of University Hospital in Ann Arbor or at Henry Ford Hospital in Detroit.

The 46 patients were studied with a protocol that included a history, physical examination, X-ray, film of the chest, and electrocardiogram. Extensive biochemical and hematologic studies were carried out, as were endocrine and immunologic testing, including lymphocyte-stimulation studies. Serum, bile, feces and fat specimens were obtained for PBB analysis. X-rays of the skull, pituitary function studies, muscle enzymes, rheumatoid factor, antinuclear antibodies, LE preparations, electromyograms and nerve conduction velocities were routinely obtained.

Patients also underwent extensive psychologic testing and psychiatric interviews. The psychologic tests included the Minnesota Multiphase Personality Inventory, Reitan-Indiana Aphasia Screening Test, Beck Depression Inventory, Wechsler Adult Intelligence Scale, H Words, Color-Naming Tests, Trail-Making Tests, Hand Dynamometer, Grooved Pegboard, Finger Tapping Test, Finger Agnosia and Dysgraphesthesia Test, Benton Facial Recognition Test, Wechsler Memory Scale and a Short-Term Memory Distractor Test. The results of these tests were compared with normal values established for age and sex differences.

\section{RESULTS}

\section{Historical Complaints}

The patients' complaints were grouped into five categories: general, musculoskeletal, skin, gastrointestinal, and neurologic. TABLE 1 lists the frequency of these findings.

\section{Physical Findings}

Complete examinations were done on all participants, and the only positive findings were the presence of hepatomegaly (72\%), skin abnormalities (28\%), objective joint abnormalities (13\%), and neurologic abnormalities (15\%). The degree of liver enlargement was mild in most cases and was not associated with tenderness or stigmata of chronic liver disease. The skin abnormalities consisted of conditions that are commonly found in the general population and there were no cases of chloracne noted. The findings in the six patients with joint abnormalities consisted of active synovitis in one patient with rheumatoid arthritis, traumatic changes in another and degenerative changes in the rest. The neurologic abnormalities consisted of sensory changes in the lower extremities. There were no abnormalities in the cranial nerves, reflexes or cerebellar testing studies. 


\section{Laboratory Findings}

Although extensive laboratory testing was undertaken, few abnormalities were found. Elevation of liver enzyme studies was the most common abnormality noted. These changes were usually minimal and only exceeded twice normal values in those patients with extensive alcohol intake. The alkaline phosphatase was normal in nine patients $(20 \%)$, lactic dehydrogenase in $10(22 \%)$, and serum transaminases (SGOT and SGPT) in three (7\%). Serum triglycerides were elevated in 14 patients $(30 \%)$, and serum iron was elevated in 12 patients $(26 \%)$. No other studies were abnormal in more than $10 \%$ of the patients. Serum PBB levels ranged from 1 to 180 parts per billion (ppb) with an average of 14 , while fat levels ranged from 30 to $34,400 \mathrm{ppb}$ with an average of 3,260 . When compared with other published studies, these patients had higher average levels. ${ }^{5}$

TABLE 1

Historical Findings in 46 PBB-Exposed Patients

\begin{tabular}{lc}
\hline Historical Finding & Number of Patients $^{*}$ \\
\hline Fatigue & $40(87)$ \\
Weakness & $30(65)$ \\
Myalgias & $25(54)$ \\
Arthralgias & $31(67)$ \\
Skin rashes & $17(37)$ \\
Frequent infections & $11(24)$ \\
Abdominal pain & $14(30)$ \\
Irregular bowel & $10(22)$ \\
Memory change & $30(65)$ \\
Decreased concentration & $28(61)$ \\
Personality change & $20(44)$ \\
Increased irritability & $32(70)$ \\
Hypersomnolence & $23(50)$ \\
Headache & $21(46)$ \\
Dizziness & $9(20)$ \\
Decreased coordination & $18(39)$ \\
Depression & $24(52)$ \\
Paresthesias & $24(52)$ \\
\hline \hline
\end{tabular}

*Figures in parentheses indicates percentage.

\section{Neurologic Studies}

Electromyograms were abnormal in six patients (13\%) with no consistent or diagnostic findings. Nerve conduction studies were abnormal in 19 patients $(41 \%)$, with slowing in sensory nerve latencies the predominant finding. The abnormal values averaged 4.7 milliseconds compared to our normal value of $<3.9$ milliseconds. There was an excellent correlation between those patients with objective findings on neurologic examination and abnormal nerve conduction studies. There was no relationship between the presence of these abnormalities and serum or fat PBB levels. 


\section{Psychologic Evaluation}

Despite the fact that an extensive battery of tests was administered, few objective abnormalities were documented. The most common findings were those of somatic preoccupation, irritability and mild depression. The tests of motor function were normal, while the tests of sensory modalities showed minor differences that were not outside normal limits. Most had I.Q.s between 100 and 140 with no differences between estimated and observed levels. Although most patients complained of memory difficulties, no objective deterioration in memory could be elicited.

Psychiatric interviews confirmed the aforementioned findings that 31 patients (67\%) were depressed. No evidence of endogenous depression was noted, and it was the opinion of the psychiatrists involved that the findings were characteristic of reactive depression. This could have resulted from the patients' material losses, the legal proceedings, their inability to obtain help, or from their multiple medical complaints which could be related to toxic effects of PBB on other organ systems.

\section{Discussion}

Many studies have been, or are now being, done to determine the health effects of PBB ingestion. Initial studies failed to document any acute changes in the health status of the subjects, and there was no relationship between the presence of symptoms and blood or fat levels of PBBs. Because of continued complaints about health, a series of follow-up studies was undertaken in an attempt to more accurately define the clinical problems. Unfortunately, most of these studies were initiated two or three years after the first contamination occurred, so that no satisfactory baseline studies were available.

A number of difficulties have befallen the farmers as a result of the contamination, and these must enter into any analysis of the health data. Among these are public charges of an initial cover-up of the entire issue, difficulty in obtaining medical care, financial ruin with the loss of livestock, and little financial aid to assist in reestablishing homesteads. Since these have the potential for increasing adverse health effects, it becomes imperative to attempt to sort out the direct effects of a toxic chemical from the behavioral problems induced by this incident and its management.

This study addresses itself to the neurobehavioral effects of PBBs and attempts to correlate them with other parameters. The study population was selected to include those patients who were previously identified as having the greatest change in health status that corresponded chronologically to the PBB contamination. It was hoped that if abnormalities truly existed, they would be most evident in this group of people who appeared to have suffered the most incapacitating effects. We did not have an appropriate control group that was matched for age, sex, and socioeconomic background, so it was necessary for us to compare our findings with normal values previously established for the tests involved.

The high prevalence of symptoms should not be surprising in view of how our patient population was selected. The similarity of these symptoms in published studies is noted. The only striking finding in the physical examination was the presence of hepatomegaly in almost three-fourths of the group. However, minimal enlargement was the rule and few abnormalities of liver function were found. In fact, the paucity of laboratory findings was remarkable when considered in light of the historical complaints. 
The electromyographic findings were not clinically useful and did not correlate with physical findings. The most common abnormality was the presence of isolated fasciculations in three patients. The nerve conduction studies revealed normal motor latency and velocity but abnormal delays in the sensory latencies. These sensory findings are often the most sensitive indications of early neuropathy, and it is not uncommon to have selective involvement of only sensory fibers similar to what is seen in diabetic neuropathy. The etiology of this is unclear, but is is possible that PBBs, with their high affinity for adipose tissue, could select our peripheral nerve tissue with its high concentration of phosphatides and sphingomyelin.

The extensive psychologic work-ups failed to identify any consistent findings aside from the presence of reactive depression. It is important to stress that despite the multiple complaints, objective evidence of organic changes in the central nervous system could not be found. Since no major changes could be identified, it is unlikely that they would be found in less exposed groups such as the public at large in the state of Michigan. The depression noted was usually better now than it was two years ago and psychopharmacologic agents have not been used in the management of these people.

We concluded that in this group of patients with known exposure to PBBs and incapacitating health care complaints, there was a high prevalence of hepatomegaly $(72 \%)$, sensory neuropathies $(41 \%)$, and reactive depression $(67 \%)$. We found no evidence of changes suggestive of organic brain syndromes and based upon historical information presented, the depressive reactions have improved over time.

These conclusions suggest that the people of the state of Michigan with little exposure to PBBs are not at great risk of suffering any adverse health effects from this exposure.

\section{REFERENCES}

1. Jackson, T. F. \& F. L. Halbert. 1974. J. Am. Vet. Med. Assoc. 165: 437.

2. ATSUKO, Y. 1971. Investigation concerning babies born from women who consumed oil contaminated with chlorobiphenyl. Fukuoka Igakkai Shi Fukuoka Acta Medica 62: 117122.

3. MasuadA, Y. 1974. Comparison of polychlorinated biphenyls in Yusho patients and ordinary persons. B. Environmental Contamination and Toxicology. Vol. II: 213-216.

4. Michigan Department of Public Health. 1975. The short term effects of PBB on health.

5. Michigan Department of Public Health. 1978. An epidemiological study of human health effects associated with the consumption of milk, meat or other foods contaminated with polybrominated biphenyl. CDC Contract \# 200-76-0625, 1978.

6. WILlETT, L. B. and H. A. IRviNG. 1975. Distribution and clearance of polybrominated bjphenyls by cows. J Dairy Sci. 58: 764.

7. VAlCIUKAS, J. A., R. LILEIS, M. S. WolfF, et al. 1978. Comparative neurobehavioral study of a PBB-exposed population in Michigan and a non-exposed group in Wisconsin. Interim Summary Report to the National Institute of Environmental Health Sciences ES 00928. 